\title{
Standard Model measurements at the High-Luminosity LHC with the CMS experiment
}

\author{
Alexander A. Savin ${ }^{* \dagger}$ \\ University of Wisconsin-Madison, Madison WI 53706-1390, USA \\ E-mail: asavin@mail.cern.ch
}

The HL-LHC project aims to achieve instantaneous luminosity a factor of five larger than the nominal LHC value. With this modification the LHC will be able to deliver $3000 \mathrm{fb}^{-1}$ of integrated luminosity over 10 years of operation, 2028 - 2038, to be compared to $300 \mathrm{fb}^{-1}$ expected to be collected by the end of 2023. A set of selected standard model measurements proposed for the CMS experiment after High-Luminosity upgrade of the LHC is discussed. The measurements are separated into three categories: precise measurements that profit from both improved systematic uncertainties and increased luminosity, like top mass and weak mixing angle measurements; measurements with low cross section that profit mainly from luminosity increase, like flavor changing neutral current in top production or VV VBS polarized cross section measurements; and measurements that profit from improved detector performance, like studying of B decays to four kaons or tau leptons to three muons.

The 39th International Conference on High Energy Physics (ICHEP2018)

4-11 July, 2018

Seoul, Korea

\footnotetext{
* Speaker.

${ }^{\dagger}$ On behalf of the CMS Collaboration
} 


\section{Introduction}

The High-Luminosity LHC (HL-LHC) relies on number of key innovative technologies, including cutting-edge 11-12 Tesla superconducting magnets, compact superconducting crab cavities with precise phase control for beam rotation, etc [1]. The CMS experiment is planning to upgrade its major parts: muon [2] and tracking [3] systems with extending pseudorapidity coverage up to $|\eta|<3$ and 3.8 respectively. Improved trigger system [4] that should use tracks and crystal granularity of the barrel calorimeter. Completely new endcap calorimeter [5]. There are many existing and new measurements that can be performed with such luminosity and improved detector capabilities, we present here only few selected proposals.

\section{Precise SM measurements}

The electroweak (EW) measurements are playing an important role at the LHC. The global standard model (SM) parameters like top mass and $\sin \theta_{W}$ are measured and provide important input to the models and global fits. The top mass is currently measured with high accuracy using different techniques, where the most precise is the "l+jets" method [6]. The comparison of results using different methods is presented in Fig. 1 left [7]. The less precise, currently, is the " $J / \Psi$ " approach that is based on a correlation between lepton from $\mathrm{W}$ decay and $J / \Psi$ from the b-quark decay in a process $t \rightarrow W b$. With increased statistics and improved systematic uncertainties it is expected that $J / \Psi$ method will significantly improve its precision and together with other methods will allow to measure the top mass with accuracy few hundreds MeV at the HL-LHC.
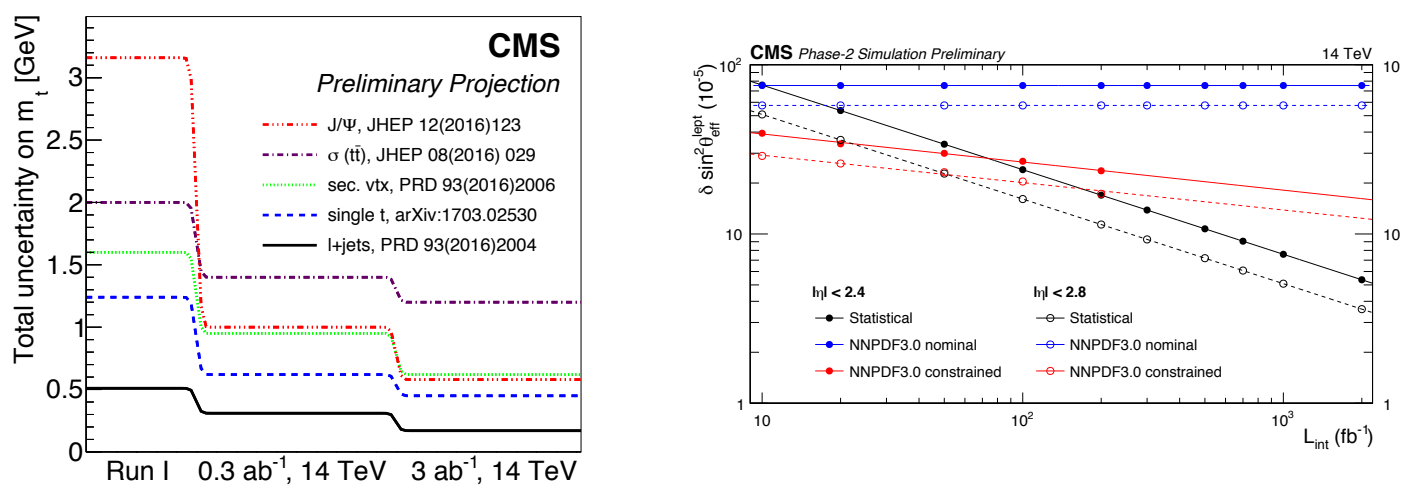

Figure 1: (left) The top mass measurement uncertainty for different methods as a function of integrated luminosity. (right) The statistical and PDF uncertainties of the weak mixing angle measurement as a function of integrated luminosity.

The presence of both vector and axial-vector couplings of electroweak bosons to fermions lead to a forward-backward asymmetry $A_{F B}$ in the production of Drell-Yan lepton pairs, that is used to extract the effective mixing angle [8]. After improvement in statistical uncertainty, as shown in Fig. 1 right, the PDF uncertainty becomes the dominating factor that restricts the precision of the measurement. This uncertainty is estimated by repeating analysis with 100 replicas of NNPDF 3.0 $\mathrm{PDF}$ and is shown in the plot as "nominal". Since the shape of the $A_{F B}$ distribution changes for 
different replicas, it results in large PDF uncertainty in the extraction of the weak mixing angle, that is performed at Z-boson mass. The effect is reduced by assigning higher weight to replicas that describe the data in the regions of low and high dimuon masses, far from the Z-boson mass, in the region that is not so sensitive to the weak-mixing-angle value. The "constrained" method not only reduces the PDF uncertainty, but also makes it luminosity dependent, since with increased luminosity the weight assignment to different replicas becomes more accurate.

\section{FCNC in top production and diboson electroweak production}

Flavor changing neutral current processes in top production, like tZq, t $\gamma q$, tgq, tHq $(q=u$, c), are forbidden at tree level and highly suppressed at high orders. The branching fractions for such processes are lying in the region $10^{-12}-10^{-16}$ and its observation above these values would immediately indicate a presence of processes beyond SM. The HL-LHC allows to significantly improve the present limits from currently measured $10^{-4}$ to $10^{-6}$ in tq $\gamma$ process for example [2].
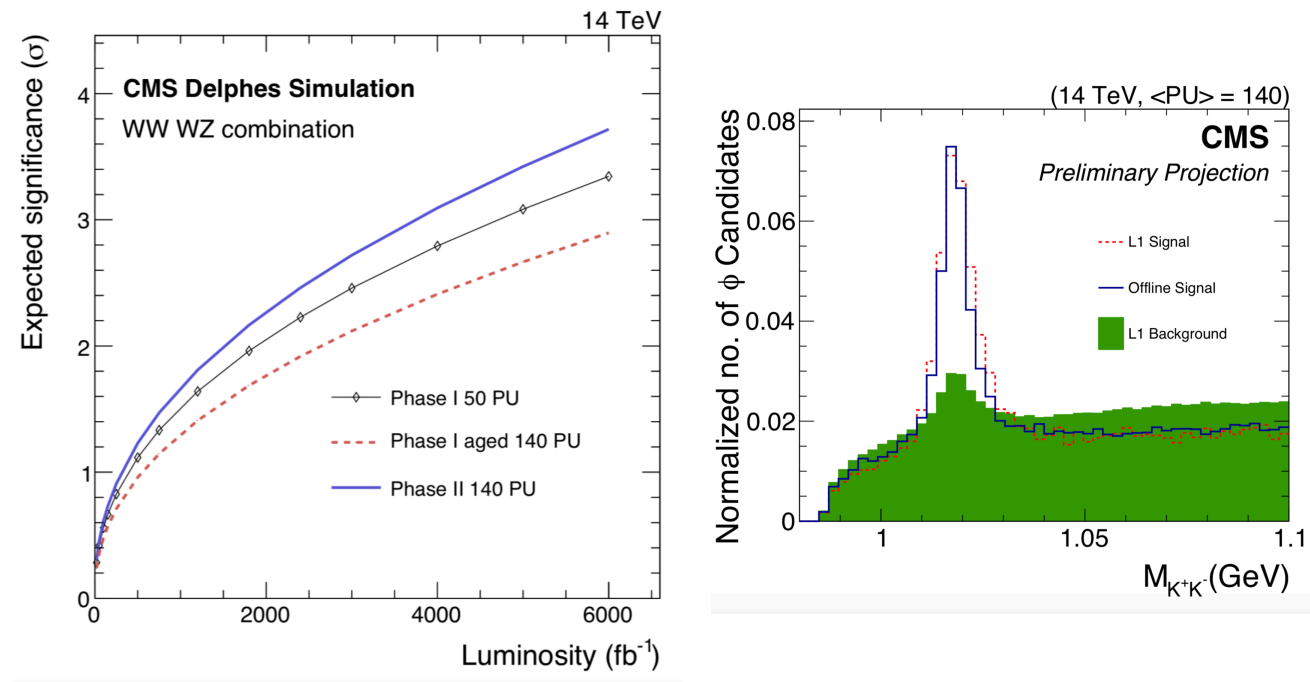

Figure 2: (left) The expected significance of the LL cross section measurement as a function of integrated luminosity. (right) The reconstructed invariant mass of two kaon candidates (tracks).

Electroweak production of vector bosons is another important measurement that requires high statistics of the data samples. This production is characterized by presence of two vector bosons, WW, WZ, or ZZ in the central part of the detector with two jets in backward/forward directions separated by a large rapidity gap. The major background to EW process is QCD production with similar signature. The total EW production is composed of three possible polarization states, when both vector bosons are longitudinally polarized (LL), and when one or both of them are polarized transversely. Figure 2 left demonstrates the expected significance of the LL cross section measurement as a function of integrated luminosity for different detector scenario [9]. It is demonstrated that by combining all channels, WW and WZ in the plot, and possibly also ZZ, the LL process will become observable at the HL-LHC. 


\section{The $B_{S}^{0} \rightarrow \phi \phi \rightarrow 4 K$ and $\tau \rightarrow 3 \mu$ decays}

The $B_{S}^{0} \rightarrow \phi \phi \rightarrow 4 K$ is another example of FCNC state that is forbidden at tree level and receives loop contribution from high masses, and can profit from new features of the upgraded CMS detector [3]. The experimental challenge of this channel is very low transverse momentum, $p_{T}$, of the outcoming kaons, down to $2-3 \mathrm{GeV}$. To select such events one needs to have a trigger that can select tracks with such a low $p_{T}$. The CMS is planning to upgrade the level 1 trigger system and to include there the tracking information. In Fig. 2 right the reconstructed invariant mass of two tracks, associated with kaons, is compared between the offline and trigger reconstruction. It is shown that the signal is very well separated from the background. The mass resolution is similar between the offline and trigger reconstructions. The efficiency of the trigger, $\approx 30 \%$, is still lower then in the offline, $\approx 55 \%$, but still sufficient to select such events for the later analysis. Thus the new trigger for CMS should allow to select such events for the physics analysis.

The lepton-flavor violating decay, $\tau \rightarrow 3 \mu$, should profit from extended pseudorapidity of the CMS muon detectors after upgrade. Since significant portion of muons will be produced in the forward direction [2] the exclusion limit for such a process will improve. The effective gain of $\approx 30 \%$ can be interpreted as an effective increase in the luminosity, e.g. $4000 \mathrm{fb}^{-1}$ instead of 3000 $\mathrm{fb}^{-1}$, or 3 additional years of the HL-LHC operation.

\section{References}

[1] HiLumi, High-Luminosity LHC project, 2018 [//hilumilhc.web.cern.ch].

[2] CMS Collaboration, The Phase-2 Upgrade of the CMS Muon Detectors, CMS-TDR-016, 2017, [//cds.cern.ch/record/2283189].

[3] CMS Collaboration, The Phase-2 Upgrade of the CMS Tracker, CMS-TDR-014, 2017, [//cds.cern.ch/record/2272264].

[4] CMS Collaboration, The Phase-2 Upgrade of the CMS L1 Trigger Interim Technical Design Report, CMS-TDR-017, 2017, [//cds.cern.ch/record/2283192].

[5] CMS Collaboration, The Phase-2 Upgrade of the CMS Endcap Calorimeter, CMS-TDR-019, 2018, [//cds.cern.ch/record/2293646].

[6] CMS Collaboration, Measurement of the top quark mass using proton-proton data at $\sqrt{s}=7$ and 8 TeV, Phys.Rev.D 932016072004.

[7] CMS Collaboration, Prospects for Standard Model measurements the HL-LHC, CMS-PAS-FTR-16-006, 2016, [//cds.cern.ch/record/2262606].

[8] CMS Collaboration, A proposal for the measurements of the weak mixing angle at the HL-LHC, CMS-PAS-FTR-17-001, 2017, [//cds.cern.ch/record/2294888].

[9] CMS Collaboration, Prospects for the study of vector boson scattering in same sign WW and WZ interactions at the HL-LHC with the upgraded CMS detector, CMS-PAS-SMP-14-008, 2016, [//cds.cern.ch/record/2220831]. 